\title{
2-(cyclooct-2-enyloxy)-isoindole-1,3-dione
}

\section{Sergiu Coseri}

Institute of Macromolecular Chemistry "P.Poni” Iasi, Gr.Ghica Voda, 41A, 6600 Iasi, Romania and National Research Council of Canada, 100 Sussex Dr., Ottawa, ON, K1A 0R6, Canada e-mail: Sergiu.Coseri@icmpp.ro

Received: 30 September 2005 / Accepted: 24 October 2005 / Published: 22 January 2006

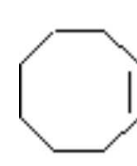<smiles>O=C1c2ccccc2C(=O)N1O</smiles>

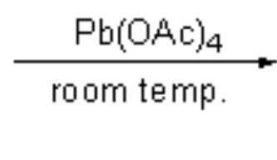<smiles>O=C1c2ccccc2C(=O)N1OC1C=CCCCCC1</smiles>

A degassed mixture containing $0.38 \mathrm{mmol}$ cyclooctene and $0.0767 \mathrm{mmol}$ N-hydroxyphthalimide (NHPI) ${ }^{1,2}$ in $5 \mathrm{~mL}$ acetonitrile was added to a degassed solution of lead tetraacetate ${ }^{3}(0.038 \mathrm{mmol}$ in 5 $\mathrm{mL}$ acetonitrile) under nitrogen atmosphere, at room temperature. After the completion of the reaction, the solvent and the olefin excess was removed under reduced pressure and the residue was purified by preparative TLC using hexane:ethyl acetate $=2: 1$ as eluent, giving the above product.

${ }^{1} \mathrm{H}-\mathrm{NMR}\left(400 \mathrm{MHz}, \mathrm{CDCl}_{3}\right)$ : $\mathrm{d}=7.84,7.77(\mathrm{Ar}, 4 \mathrm{H}) ;$ 5.86, $5.77(\mathrm{CH}=\mathrm{CH}, 2 \mathrm{H}) ; 5.32(\mathrm{O}-\mathrm{CH}, 1 \mathrm{H}) ; 2.22$, 2.10, 1.67, 1.61, $1.59\left(\mathrm{CH}_{2}, 10 \mathrm{H}\right)$.

${ }^{13} \mathrm{C}-\mathrm{NMR}\left(100 \mathrm{MHz}, \mathrm{CDCl}_{3}\right): \mathrm{d}=164.6(\mathbf{C}=\mathrm{O}) ; 134.9,128.2(\mathrm{Ar}) ; 85.7(\mathbf{C}-\mathrm{O}) ; 77.6(\mathbf{C}=\mathbf{C}) ; 33.7,30.5$, 28.7, 26.7, $24.6\left(\mathrm{CH}_{2}\right)$.

\section{References}

1. Ishii, Y.; Sakaguchi, S.; Iwahama, T., Adv. Synth. Catal., 2001, 343, 393.

2. Masui, M.; Hosomi, K.; Tsuchida, K.; Ozaki, S., Chem. Pharm. Bull., 1985, 33, 4798.

3. Martin, J.C.; Taylor, J.W.; Drew, E.H., JACS, 1967, 89, 129.

Sample Availability: Available from MDPI.

(C) 2006 MDPI. All rights reserved. 